\title{
Produção de carvão ativado microporoso com vapor d'água preparado a partir de endocarpo de coco (Cocos nucifera)
}

\author{
Rayssa de Medeiros Morais ${ }^{1 *}$ Gregório Mateus Santana ${ }^{1}$ Roberto Carlos Costa Lelis ${ }^{1}$ Juarez Benigno Paes ${ }^{2}$ \\ ${ }^{1}$ Universidade Federal Rural do Rio de Janeiro, Rodovia BR 465, Km07, s/n Zona Rural, Seropédica - RJ, 23890-000 \\ ${ }^{2}$ Universidade Federal do Espírito Santo, Alto Universitário, S/N Guararema, Alegre - ES, 29500-000 \\ *Author for correspondence: rayssaengflorestal @ gmail.com
}

Received: May 2018 / Accepted: August 2019/ Published: September 2019

\section{Resumo}

Este estudo teve por objetivo produzir carvão ativado (CA) a partir de endocarpo de coco (Cocos nucifera) e aplicá-lo para adsorção do corante azul de metileno em meio aquoso. O CA foi preparado mediante ativação física, utilizando como agente ativante o vapor d'água. Em seguida, realizadas caracterizações relativas ao rendimento gravimétrico em carvão ativado $\left(\mathrm{RG}_{\mathrm{CA}}\right)$, análise termogravimétrica, área superficial ( $\left.\mathrm{S}_{\mathrm{BET}}\right)$, volume e diâmetro dos poros e microscopia eletrônica de varredura. Para avaliação da capacidade adsortiva, foram realizados estudos de cinética e isotermas de adsorção, e aplicados os modelos de isotermas de Langmuir e de Freundlich. O CA produzido apresentou $\mathrm{RG}_{\mathrm{CA}}$ de $20,69 \%$,

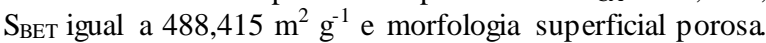
Apresentou uma capacidade máxima de adsorção $\left(\mathrm{q}_{\mathrm{m}}\right)$ para o corante azul de metileno igual a 203,04 $\mathrm{mg} \mathrm{g}^{-1}$, ajustando-se melhor ao modelo de Freundlich. O CA produzido apresentou excelentes propriedades (texturais e adsortivas), sendo eficiente para adsorção do corante azul de metileno.

Palavras-chave: Adsorventes de baixo custo; aplicação ambiental; ativação física.

\section{Abstract}

This study had as an objective to produce activated carbon (AC) from coconut shell (Cocos nucifera) and apply this activated carbon to adsorption of methylene blue dye in aqueous medium. AC was prepared by physical activation, using water steam as the activating agent. Then, we realized characterizations related to the gravimetric yield in activated carbon $\left(\mathrm{GY}_{\mathrm{AC}}\right)$, thermogravimetric analysis, surface area $\left(\mathrm{S}_{\mathrm{BET}}\right)$, pore volume and diameter and scanning electron microscopy. The adsorption capacity was evaluated from kinetic studies and adsorption isotherms and Langmuir and Freundlich isotherm models were applied. The AC produced had $20.69 \% \mathrm{GY}_{\mathrm{AC}}, \mathrm{S}_{\mathrm{BET}}$ equal to $488.415 \mathrm{~m}^{2} \mathrm{~g}^{-1}$ and porous surface morphology. The AC presented a maximum adsorption capacity $\left(\mathrm{q}_{\mathrm{m}}\right)$ for the methylene blue dye equal to $203.04 \mathrm{mg} \mathrm{g}^{-1}$, adjusting better to the Freundlich model. The AC had excellent properties (textural and adsorptive), being efficient for adsorption of methylene blue dye.

Keywords: Low-cost adsorbents; environmental applications; physical activation.

\section{Introdução}

O fornecimento de água potável é uma das questões mais preocupantes em todo o mundo por causa do contínuo desenvolvimento econômico e do aumento constante da população mundial. Neste sentido, as fontes de água potável estão diminuindo todos os dias, devido à contaminação por vários poluentes, incluindo produtos químicos orgânicos, a exemplo dos produtos farmacêuticos, pesticidas, corantes e compostos fenólicos, todos poluentes nocivos que devem ser removidos dos corpos d' água (Hasan e Jhung 2015).
A técnica de adsorção por carvão ativado é empregada para remover esses poluentes que são lançados nos corpos d'água, e dessa forma, cada vez mais os pesquisadores têmse concentrado no desenvolvimento de materiais obtidos a partir de precursores de baixo custo e renováveis em substituição aos materiais minerais (Wong et al. 2018).

Os métodos de ativação e a natureza do material precursor influenciam na formação dos grupos funcionais superficiais e na estrutura porosa do carvão ativado. Deste modo, o foco das pesquisas têm sido desenvolver ou modificar as técnicas de ativação existentes usando precursores apropriados para poluentes específicos (Bhatnagar et al. 2013).

No beneficiamento do coco seco, após extrair o produto principal, que é a polpa ou endosperma, resta uma quantidade significativa de resíduo. O mesocarpo fibroso possui fibras longas usadas na fabricação de mantas, tapetes, fibra para colchões, sendo o pó gerado no processamento usado também como substrato agrícola. O endocarpo, parte mais rígida e escura, é um combustível lenhoso com alto poder calorífico, além de ser um excelente precursor para produção de carvão ativado (Fontenele 2005; Mangueira 2014).

Diante do contexto, este estudo teve por objetivo produzir e avaliar a qualidade do carvão ativado obtido a partir de endocarpo de coco (Cocos nucifera) e ap licá-lo para adsorção do corante azul de metileno presente em meio aquoso.

\section{Material e Métodos \\ Produção do carvão ativado}

$\mathrm{O}$ endocarpo de coco foi obtido do comércio local da cidade de João Pessoa, Paraíba. As amostras foram secas ao ar, trituradas em moinho de martelo e classificadas em peneiras vibratórias. Antes de serem trituradas houve remoção dos resquícios da amêndoa e das fibras que envolvem a parte externa do endocarpo. As frações utilizadas na produção do CA foram as que passaram na peneira de 8 mesh e ficaram retida na peneira de 30 mesh.

Para produção do $\mathrm{CA}$ foi empregado o processo de ativação física direta, ou seja, o material precursor foi colocado "in natura" no forno de ativação, eliminando a etapa de carbonização, comumente empregada, sendo ainda usado como agente ativante o vap or d'água, em uma planta de forno semi-industrial, com um forno rotativo da CHINO, marca Takabayashi Rica, modelo RS-S, Japão. Para o processo de ativação utilizou-se $100 \mathrm{~g}$ de material precursor que foram inseridas em um reator tubular de leito fixo, com dimensões de $93 \mathrm{~mm}$ de diâmetro e $900 \mathrm{~mm}$ de comprimento. Ao forno estão acoplados uma caldeira elétrica para o fornecimento do vapor d'água e um recipiente "dewar" para nitrogênio $\left(\mathrm{N}_{2}\right)$.

Foi empregada uma taxa de aquecimento de $10{ }^{\circ} \mathrm{C} \mathrm{min}^{-1}$, que saiu de $25^{\circ} \mathrm{C}$ para $800{ }^{\circ} \mathrm{C}$, sob atmosfera inerte de $\mathrm{N}_{2}$ (fluxo de $80 \mathrm{~mL} \mathrm{~min}^{-1}$ ) e tempo de residência na temperatura final de 30 minutos. Na temperatura final, foi introduzido o vapor d'água com fluxo controlado de $100 \mathrm{~mL} \mathrm{~min}^{-1}$. O CA obtido a partir de endocarpo de coco foi denominado CAC. 
Morais et al.

Rendimento gravimétrico em Carvão ativado (RGCA)

$\mathrm{O}$ rendimento gravimétrico em $\mathrm{CA}\left(\mathrm{RG}_{\mathrm{CA}}\right)$ foi calculado de acordo com a Equação 1:

$\mathrm{RG}_{\mathrm{CA}}(\%)=\frac{\mathrm{m}_{\mathrm{f}}}{\mathrm{m}_{0}} \times 100$

onde: $\mathrm{m}_{\mathrm{f}}=$ massa seca final do $\mathrm{CA}(\mathrm{g}) ; \mathrm{m}_{0}=$ massa seca inicial do material precursor $(\mathrm{g})$.

Análise termogravimétrica, área superficial e a porosidade

Para análise termogravimétrica utilizou-se uma balança termogravimétrica Q50 da marca TA Instruments, com um fluxo de $50 \mathrm{~mL} \mathrm{~min}^{-1}$ de $\mathrm{N}_{2}$, massa da amostra de $10 \mathrm{mg}$ que foi colocada em um cadinho de platina, usando uma taxa de $10{ }^{\circ} \mathrm{C} \mathrm{mim}{ }^{-1}$ até a temperatura final de $900{ }^{\circ} \mathrm{C}$.

A área superficial, volume e diâmetro dos poros foram determinadas através da análise BET (Brunauer, Emmett e Teller), que utiliza a técnica de adsorção/dessorção de $\mathrm{N}_{2}$, a $77 \mathrm{~K}$, empregando um Microporosímetro, modelo ASAP 2020, marca Micromeritics, conectado a um microcomputador. As amostras de CAC $(0,25 \mathrm{~g})$ foram antes desgaseificada a vácuo a $300{ }^{\circ} \mathrm{C}$, para eliminar a umidade e outros possíveis gases presentes (Skaar 1988).

\section{Microscopia eletrônica de varredura}

A morfologia superficial foi obtida através de análise de Microscopia Eletrônica de Varredura (MEV), realizada em um aparelho FEI Quanta 450, ao empregar tensão de 20 kv. As amostras foram montadas sobre plataforma de alumínio, com o uso de fita de carbono dupla com uma fina camada de ouro, depositada por um metalizador Emitech k550X.

\section{Cinética de adsorção}

Estudos de cinética de adsorção foram realizados em temperatura ambiente, sendo utilizados $10 \mathrm{mg}$ de CA e $10 \mathrm{~mL}$ de solução com o corante na concentração de $50 \mathrm{mg} \mathrm{L}^{-1}$. Em intervalos predeterminados de 1,2, 3, 6, 12 e 24 horas, alíquota da solução foram retiradas e suas concentrações determinadas. Para a determinação da concentração de equilíbrio foi utilizado um espectrofotômetro, modelo UVmini 1240 , da SHIMADZU, com o comprimento de onda $(\lambda)$ de $665 \mathrm{~nm}$ característico para o azul de metileno.

\section{Isotermas de adsorção}

As isotermas de adsorção foram obtidas ao serem empregadas $10 \mathrm{mg}$ de adsorvente e $10 \mathrm{~mL}$ da solução com diferentes concentrações do corante azul de metileno, que foram inseridas em recipientes plásticos de $20 \mathrm{~mL}$ e mantidos sob agitação de $100 \mathrm{rpm}$ em uma incubadora Shaker (modelo SL 221, da SOLAB) por 24 horas, à temperatura ambiente.

Foram preparadas curvas de calibração com soluções de concentrações de 25, 50, 100, 250, 500 e $1000 \mathrm{mg} \mathrm{L}^{-1}$ para o corante azul de metileno. A quantidade de adsorvato adsorvido, $\mathrm{q}_{\mathrm{eq}}$, por grama de adsorvente, foi calculada de acordo com a Equação (2).

$q_{e q} \frac{\left(c_{0}-C_{e q}\right) x V}{m}$

onde: $\mathrm{C}_{0}$ : concentração inicial do corante azul de metileno (mg L $\left.{ }^{-1}\right) ; \mathrm{C}_{\mathrm{eq}}$ : concentração do corante azul de metileno após atingir o equilíbrio de adsorção $\left(\mathrm{mg} \mathrm{L}^{-1}\right)$; V: volume de adsorvato (L); m: massa de adsorvente (g).

\section{Modelos utilizados para os ajustes das isotermas obtidas}

As isotermas de adsorção foram ajustadas de acordo com os modelos de Langmuir e Freundlich, descritos na Tabela 1, onde $\mathrm{q}_{\mathrm{eq}}$ é a quantidade de adsorvato adsorvida por grama de adsorvente no equilíbrio ( $\left.\mathrm{mg} \mathrm{g}^{-1}\right)$; $\mathrm{C}_{\mathrm{eq}}$ é a concentração do adsorvato após atingido o equilíbrio $\left(\mathrm{mg} \mathrm{L}^{-1}\right)$; $\mathrm{qm}_{\mathrm{m}}$ é a capacidade máxima de adsorção $\left(\mathrm{mg} \mathrm{g}^{-1}\right) ; \mathrm{K}_{\mathrm{L}}$ a constante de Langmuir $\left(\mathrm{L} \mathrm{mg}^{-1}\right)$; e $\mathrm{K}_{\mathrm{F}}$ o coeficiente de sorção $\left[\left(\mathrm{mg} \mathrm{L}^{-1}\right)(\mathrm{L}\right.$ $\left.\mathrm{mg}^{-1}\right)^{1 / n}$ ] e $1 / \mathrm{n}$ a medida da intensidade de sorção.

Tabela 1. Princípios dos modelos de Langmuir e Freundlich.

\begin{tabular}{ccc}
\hline Modelo & Equação & Equação Linearizada \\
\hline Langmuir & $q_{e q}=\frac{q_{m} K_{L} C_{e q}}{1+K_{L} C_{e q}}$ & $\frac{C_{e q}}{q_{e q}}=\frac{1}{q_{m} K_{L}}+\frac{1}{K_{L}} C_{e q}$ \\
\hline Freundlich & $q_{e q}=K_{F} C_{e q}^{1 / n}$ & $\log q_{e q}=\log K_{F}+\frac{1}{n} \log C_{e q}$ \\
\hline
\end{tabular}

\section{Resultados e discussão}

\section{Rendimento gravimétrico em carvão ativado}

O CAC apresentou $R_{C A}$ de 20,69\%, sendo estável termicamente, que pode estar associada à composição química do precursor, principalmente em relação ao teor de lignina, pois, em geral, materiais com grande quantidade deste componente, apresentam uma melhor estabilidade térmica e consequentemente maiores rendimentos (Reed e Williams 2004). Na literatura, o teor de lignina para o endocarpo de coco é cerca de 33\% (Arena et al. 2016).

\section{Análise termogravimétrica}

Nas Figuras de 1 e 2 são apresentados os termogramas de decomposição térmica do precursor e do CAC. As curvas de TG e DTG para o CAC foram obtidas afim de monitorar a estabilidade térmica do material após a ativação, e também avaliar em que temperatura proceder a regeneração do CAC após a sua saturação. A degradação ocorre em temperaturas inferiores a $100{ }^{\circ} \mathrm{C}$, sendo essa primeira perda de massa atribuída a perda de umidade dos materiais. Após esse primeiro evento, o CAC manteve-se relativamente estável até uma temperatura em torno de $700{ }^{\circ} \mathrm{C}$, quando começa a perder massa, visto que a ativação foi realizada na temperatura de $800{ }^{\circ} \mathrm{C}$. Dessa forma, o CAC apresentou uma boa estabilidade térmica, podendo ser regenerado até temperaturas próximas a temperatura de ativação, sem que haja grandes perdas em massa do material a ser regenerado.

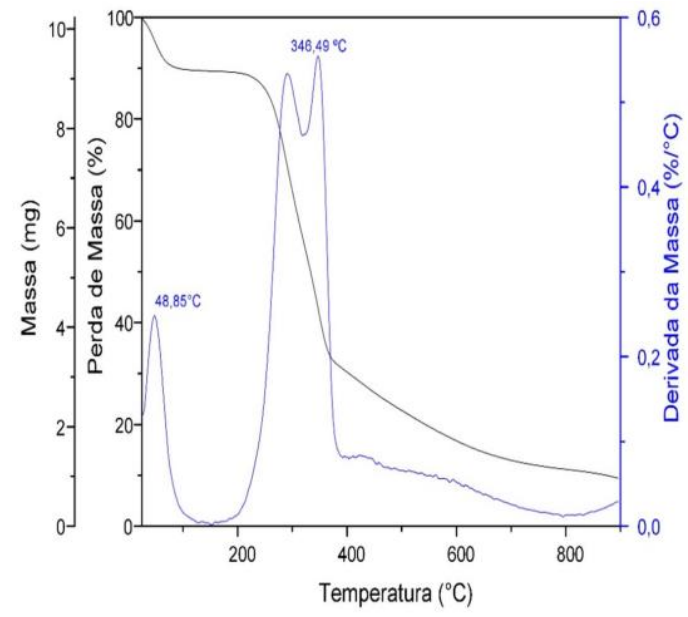

Figura 1. Curvas de TG e DTG para o endocarpo do coco. 
Morais et al.

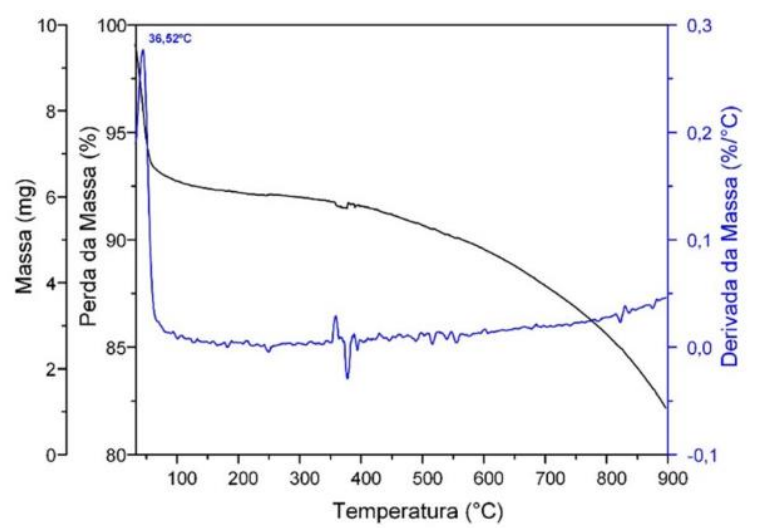

Figura 2. Curvas de TG e DTG para o carvão ativado de endocarpo do coco.

\section{Área superficial e a porosidade}

A forma das isotermas podem fornecer informações qualitativas preliminares sobre o mecanismo de adsorção e da estrutura porosa do CAC (Brum et al. 2008). Na Figura 3, observa-se que a isoterma do CAC apresenta elevada adsorção de $\mathrm{N}_{2}$ a baixas pressões relativas $\left(\mathrm{P} / \mathrm{P}_{0}<1\right)$, o que indica a natureza microporosa do material (Couto et al. 2012).

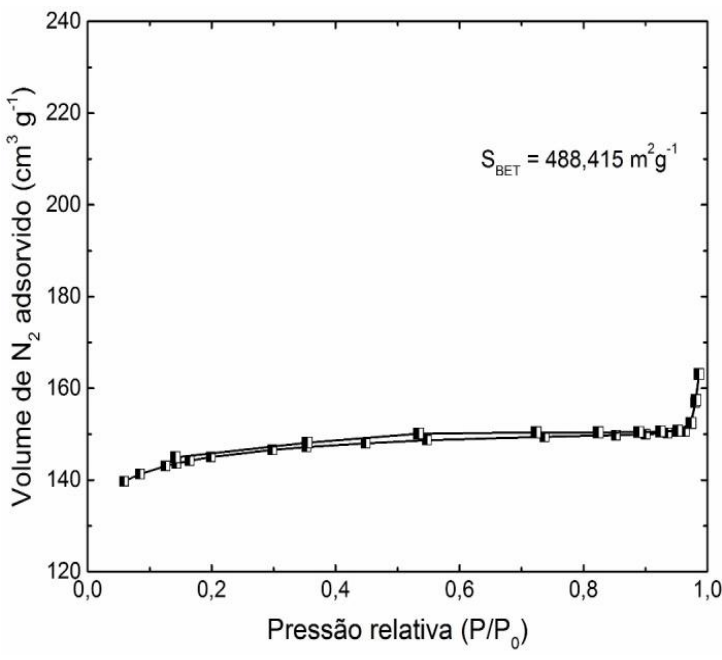

Figura 3. Isotermas de adsorção/dessorção de $\mathrm{N}_{2}$, a $77 \mathrm{~K}$, para o CAC.

Em resumo, por análise de adsorção/dessorção de $\mathrm{N}_{2}$, os valores obtidos relativos às propriedades texturais do CAC obtido são apresentados na Tabela 2.

Tabela 2. Propriedades texturais obtidas por análise BET.

\begin{tabular}{cc}
\hline Análise B ET & CAC \\
\hline Área superficial BET $\left(\mathrm{S}_{\mathrm{BET}}\right)$ & $488,415 \mathrm{~m}^{2} \mathrm{~g}^{-1}$ \\
Área superficial de microporos $\left(\mathrm{S}_{\mathrm{M}}\right)$ & $417,398 \mathrm{~m}^{2} \mathrm{~g}^{-1}$ \\
Área superficial externa ( $\left.\mathrm{S}_{\mathrm{ext} .}\right)$ & $71,017 \mathrm{~m}^{2} \mathrm{~g}^{-1}$ \\
Volume total de poros ( $\left.\mathrm{V}_{\mathrm{P}}\right)$ & $0,244 \mathrm{~cm}^{3} \mathrm{~g}^{-1}$ \\
Volume de microporos $\left(\mathrm{V}_{\mathrm{M}}\right)$ & $0,193 \mathrm{~cm}^{3} \mathrm{~g}^{-1}$ \\
Volume de mesoporos $\left(\mathrm{V}_{\mathrm{MS}}\right)$ & $0,038 \mathrm{~cm}^{3} \mathrm{~g}^{-1}$ \\
Diâmetro médio dos mesmos (D) & $19,948 \AA$
\end{tabular}

Observa-se que o material produzido é predominantemente microporoso, haja vista que $85,5 \%$ da área superficial $\mathrm{S}_{\mathrm{BET}}$ é constituída de microporos, confirmando a análise preliminar demonstrada na Figura 3. Logo, a ativação física, empregando vapor d'água, nas condições realizadas, para o precursor analisado, propiciou um incremento da porosidade e área superficial, a qual foi semelhante, por vezes superior a materiais ativados a partir de outros materiais da literatura (Tabela 3 ).

Tabela 3. Área superficial $\mathrm{S}_{\mathrm{BET}}$ por diferentes materiais precursores

\begin{tabular}{|c|c|c|c|c|}
\hline $\begin{array}{l}\text { Material } \\
\text { precursor }\end{array}$ & $\begin{array}{c}\text { Tipo de } \\
\text { ativação }\end{array}$ & $\begin{array}{l}\text { Temp. e tempo } \\
\text { de ativação }\end{array}$ & $\underset{\left(\mathbf{m}^{2} \mathbf{g}-{ }^{1}\right)}{\left.S_{\text {BET }}\right)}$ & Autores \\
\hline $\begin{array}{l}\text { Endocarpo de } \\
\text { coco }\end{array}$ & $\begin{array}{c}\text { Física com } \\
\text { vapor d'água }\end{array}$ & $800^{\circ} \mathrm{C} / 0,5 \mathrm{~h}$ & 488,415 & $\begin{array}{l}\text { Este } \\
\text { trabalho }\end{array}$ \\
\hline $\begin{array}{l}\text { Casca de } \\
\text { nozes }\end{array}$ & $\begin{array}{l}\text { Química com } \\
\mathrm{ZnCl}_{2}\end{array}$ & $600^{\circ} \mathrm{C} / 2 \mathrm{~h}$ & 427,00 & $\begin{array}{c}\text { Costa et al. } \\
(2015)\end{array}$ \\
\hline $\begin{array}{l}\text { Sabugo de } \\
\text { milho }\end{array}$ & $\begin{array}{l}\text { Química com } \\
\mathrm{ZnCl}_{2}\end{array}$ & $500^{\circ} \mathrm{C} / 3 \mathrm{~h}$ & 501,00 & $\begin{array}{l}\text { Sales et al. } \\
\text { (2015) }\end{array}$ \\
\hline $\begin{array}{l}\text { Resíduos de } \\
\text { Apuleia } \\
\text { leiocarpa }\end{array}$ & $\begin{array}{l}\text { Física com } \\
\qquad \mathrm{CO}_{2}\end{array}$ & $850^{\circ} \mathrm{C} / 1 \mathrm{~h}$ & 564,90 & $\begin{array}{c}\text { Nobre et al. } \\
(2015)\end{array}$ \\
\hline $\begin{array}{l}\text { Casca de } \\
\text { Nozes }\end{array}$ & $\begin{array}{l}\text { Química com } \\
\mathrm{ZnCl}_{2}\end{array}$ & $600^{\circ} \mathrm{C} / 2 \mathrm{~h}$ & 542,00 & $\begin{array}{c}\text { Costa et al. } \\
(2015)\end{array}$ \\
\hline $\begin{array}{l}\text { Pecíolo de } \\
\text { Palmeira }\end{array}$ & $\begin{array}{c}\text { Física com } \\
\mathrm{CO}_{2}\end{array}$ & $850^{\circ} \mathrm{C} / 0,5 \mathrm{~h}$ & 546,00 & $\begin{array}{l}\text { Rezma et al. } \\
\text { (2017) }\end{array}$ \\
\hline Candeia & $\begin{array}{l}\text { Física com } \\
\mathrm{CO}_{2}\end{array}$ & $900^{\circ} \mathrm{C} / 1 \mathrm{~h}$ & 364,00 & $\begin{array}{l}\text { Borges et al. } \\
\quad(2016)\end{array}$ \\
\hline
\end{tabular}

\section{Microscopia eletrônica de varredura}

A Figura 4 apresenta uma micrografia do CAC produzido, sendo possível verificar a estrutura porosa do material, composto por poros de tamanhos e formas uniformes (Figura 4a), distribuídos de maneira espaçada pela superfície do material (Figura 4b).
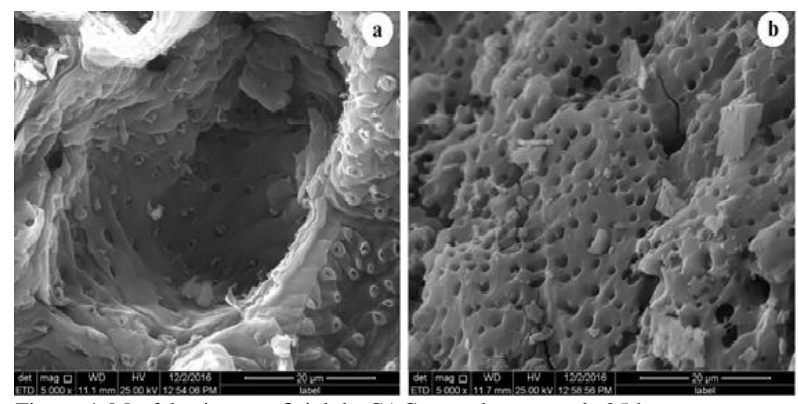

Figura 4. Morfologia superficial do CAC, usando tensão de 25 kv.

Cinética de adsorção do azul de metileno sobre o CAC

Observa-se uma rápida diminuição na concentração do corante azul de metileno durante as primeiras horas de reação (Figura 5). Em 6 horas, mais de 50\% do corante já havia sido adsorvido e com 24 horas de reação já estava próximo basicamente ao equilíbrio de adsorção, sendo praticamente todos os sítios ativos dos CAC já preenchidos pelo corante. 
Morais et al.

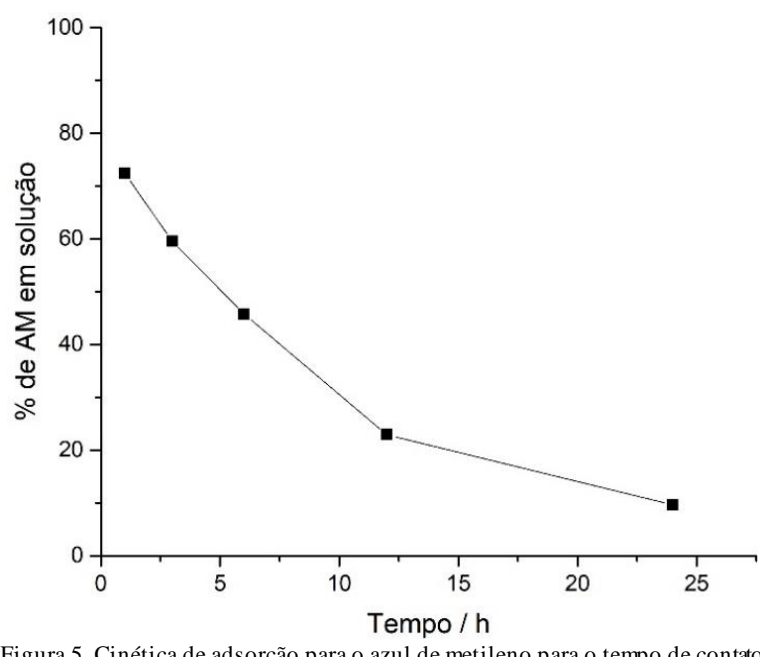

Figura 5. Cinética de adsorção para o azul de metileno para o tempo de contato adsorvato/adsorvente (10 mg de CA; $10 \mathrm{~mL}$ de solução $\left.50 \mathrm{mg} \mathrm{L}^{-1} ; 25^{\circ} \mathrm{C}\right)$.

\section{Isoterma de Adsorção}

Os parâmetros obtidos para adsorção do azul de metileno utilizando soluções com diferentes concentrações do corante analisado foi apresentada na Tabela 4 , sendo os valores ajustados de acordo com os modelos de isotermas de Langmuir e de Freundlich.

\begin{tabular}{llllllll}
\multicolumn{1}{c}{ Tabela 4.P arâmetros de Langmuir obtidos para adsorção de azul de metileno } \\
Adsorvente & \multicolumn{3}{c}{$\begin{array}{c}\text { Parâmetros de } \\
\text { Langmuir }\end{array}$} & \multicolumn{4}{c}{$\begin{array}{c}\text { Parâmetros de } \\
\text { Freundlich }\end{array}$} \\
\cline { 2 - 7 } & & $\mathbf{q}_{\mathbf{m}}$ & $\mathbf{K}_{\mathbf{F}}$ & $\mathbf{1} / \mathbf{n}$ & $\mathbf{R}^{2}$ & $\mathbf{k}_{\mathbf{L}}$ & $\mathbf{R}^{2}$ \\
\hline CAC & 203,04 & 45,11 & 0,24 & 0,928 & 0,060 & 0,909
\end{tabular}

$\mathrm{q}_{\mathrm{m}}=$ quantidade máxima de adsorção $\left(\mathrm{mg} \mathrm{g}^{-1}\right) ; \mathrm{K}_{\mathrm{L}}=$ constante de Langmuir $\left(\mathrm{L} \mathrm{mg}^{-1}\right) ; \mathrm{K}_{\mathrm{F}}=$ constante de Freundlich $\left(\mathrm{mg} \mathrm{g}^{-1}\right)\left(\mathrm{L} \mathrm{g}^{-1}\right)^{1 / n} ; 1 / \mathrm{n}=$ parâmetro de Freundlich; e $\mathrm{R}^{2}=$ coeficiente de correlação.

O CAC apresentou capacidade máxima $\left(\mathrm{q}_{\mathrm{m}}\right)$ de adsorção para o AM de 203,04 $\mathrm{mg} \mathrm{g}^{-1}$ e melhor ajuste ao modelo de Freundlich $\left(\mathrm{R}^{2}=0,928\right)$. Os valores obtidos foram comparados com os resultados encontrados para CAs preparados a partir de diferentes precursores da literatura, conforme Tabela 5. Os resultados permitem inferir que o CAC obteve elevada capacidade de adsorção para o azul de metileno, o que o torna promissor para o processo de tratamento de corpos d'água contaminados por esse corante.

Tabela 5. Capacidade máxima de adsorção $\left(\mathrm{q}_{\mathrm{m}}\right)$ de azul de metileno por vários adsorventes.

\begin{tabular}{ccc}
\hline Material precursor & $\mathbf{q}_{\mathbf{m}}\left(\mathbf{m g ~ g}^{-\mathbf{1}}\right)$ & Ref erência \\
\hline Endocarpo de coco & 203,04 & Este trabalho \\
Eucalyptus sp. & 176,46 & Couto et al. (2012) \\
sabugo de milho & 124,00 & Sales et al. (2015) \\
Candeia & 324,50 & Borges et al. (2015) \\
Pongamia pinnata & 154.8 & Islam et al. (2017) \\
\hline
\end{tabular}

\section{Conclusões}

O precursor estudado, endocarpo de coco, produziu um carvão ativado de excelente qualidade, que pode ser utilizado como adsorvente de corantes em meio aquoso, tendo o mesmo apresentando $\mathrm{S}_{\mathrm{BET}}=488,415 \mathrm{~m}^{2} \mathrm{~g}^{-1}$ e $\mathrm{q}_{\mathrm{m}}=$
203,04 $\mathrm{mg} \mathrm{g}^{-1}$, além de rápida velocidade de adsorção do corante, ajustando-se melhor ao modelo de Freundlich.

\section{Referências}

Arena N, Lee J, Clift R (2016) Life Cycle Assessment of activated carbon production from coconut shells. Journal of Cleaner Production, 125: 68-77. doi: 10.1016/j.jclepro.2016.03.073.

Bhatnagar A, Hogland W, Marques M, Sillanpää M (2013) An overview of the modification methods of activated carbon for its water treatment applications. Chemical Engineering Journal, 219: 499-511. doi: 10.1016/j.cej.2012.12.038.

Borges WMS, Arantes ACC, Bianchi ML, Resende EC, Boari PV, Guerreiro MC, Perígolo DM (2015) Carvão Ativado de Resíduo de Madeira de Candeia: Produção, Caracterização e Avaliação do Potencial Adsortivo. Revista Virtual de Química, 7(6): 1952-1967. doi: 10.5935/19846835.20150115 .

Borges WMS, Arantes ACC, Castro GMM, Bianchi ML, Nobre JRC, Resende EC, Castro JP, Guerreiro MC (2016) Produção, caracterização e avaliação da capacidade adsortiva de carvões ativado em forma de briquete. Revista Matéria, 21(4): 930 - 942. doi: 10.1590/S1517707620160004.0086 .

Brum SS, Bianchi ML, Silva VD, Gonçalves M, Guerreiro MC, Oliveira LCA (2008) Preparação e caracterização de carvão ativado produzido a partir de resíduos do beneficiamento do café. Quimica Nova, 31(5): 1048-1052.

Costa PD, Furmanski LM, Dominguini L (2015) Produção, Caracterização e Aplicação de Carvão Ativado de Casca de Nozes para Adsorção de Azul de Metileno. Revista Virtual de Química, 7(4): 1272-1285. doi: 10.5935/19846835.20150070.

Couto GM, Dessimoni ALDA, Bianchi ML, Perígolo DM, Trugilho PF (2012) Use of sawdust Eucalyptus sp. in the preparation of activated carbons. Ciência e Agrotecnologia, 36(1): 69-77, doi: 10.1590/S1413-70542012000100009.

Fontenele RES (2005) Cultura do coco no Brasil: caracterização do mercado atual e perspectivas futuras. In: Congresso da Sober, Ribeirão Preto, Brasil.

Hasan Z, Jhung SH (2015) Removal of hazardous organics from water using metal-organic frameworks (MOFs): plausible mechanisms for selective adsorptions. Journal of hazardous materials, 283: 329-339. doi: 10.1016/j.jhazmat.2014.09.046.

Islam MA, Sabar S, Benhouria A, Khanday WA, Asif M, Hameed BH (2017) Nanoporous activated carbon prepared from karanj (Pongamia pinnata) fruit hulls for methylene blue adsorption. Journal of the Taiwan Institute of Chemical Engineers, 74: 96-04. doi: 10.1016/j.je20.20.01.016.

Mangueira ESV (2014) Produção de carvão ativado a partir de endocarpo de coco da baía (Cocos nucifera) aplicado ao processo de adsorção do herbicida metribuzin. Dissertação, Universidade Federal da Paraíba. 103p. 
Morais et al.

Nobre JCR, Paz Castro J, Bianchi ML, Silva Borges WM, Trugilho PF, Cabral Moulin J, Napoli A (2015) Produção de carvão ativado de resíduo madeireiro da região Amazônica. Scientia Forestalis, 43(108): 895-906. doi: 10.18671/scifor.v43n108.14.

Reed AR, Williams PT (2004) Thermal processing of biomass natural fibre wastes by pyrolysis. International Journal of Energy Research, 28(2): 131-145. doi: 10.1002/er.956.

Rezma S, Birot M, Hafiane A, Deleuze H (2017) Phy sically activated microporous carbon from a new biomass source: Date palm petioles. Comptes Rendus Chimie, 20(9-10): 881-887. doi: 10.1016/j.crci.2017.05.003.

Sales PF, Bertoli AC, Pinto FM, Magriotis ZM (2015) Produção, Caracterização e Aplicação do Carvão Ativado Obtido a partir do Sabugo de Milho: A Busca pelo Reaproveitamento de um Resíduo Agroindustrial. Revista Virtual de Química, 7(4): 1174-1188. doi: 10.5935/19846835.20150066 .

Skaar C (1988). Wood-water relations. New York: Springer (Springer Series in Wood Science).

Wong S, Ngadi N, Inuwa IM, Hassan O (2018) Recent advances in applications of activated carbon from biowaste for wastewater treatment: A short review. Journal of Cleaner Production, 175: 361-375. doi: 10.1016/j.jclepro.2017.12.059. 